\title{
Convergence analysis of the scaled boundary finite element method for the Laplace equation
}

\author{
Fleurianne Bertrand ${ }^{1,2,3}$. Daniele Boffi, ${ }^{4,5}$. Gonzalo G. de Diego ${ }^{6}$ (D)
}

Received: 4 May 2020 / Accepted: 8 February 2021 / Published online: 19 April 2021

(C) The Author(s) 2021

\begin{abstract}
The scaled boundary finite element method (SBFEM) is a relatively recent boundary element method that allows the approximation of solutions to partial differential equations (PDEs) without the need of a fundamental solution. A theoretical framework for the convergence analysis of SBFEM is proposed here. This is achieved by defining a space of semi-discrete functions and constructing an interpolation operator onto this space. We prove error estimates for this interpolation operator and show that optimal convergence to the solution can be obtained in SBFEM. These theoretical results are backed by two numerical examples.
\end{abstract}

Keywords Scaled boundary finite element method · Error analysis ·

Singular solutions

Mathematics Subject Classification (2010) $65 \mathrm{~N} 12 \cdot 65 \mathrm{~N} 15 \cdot 65 \mathrm{~N} 30 \cdot 65 \mathrm{~N} 38$

\section{Introduction}

The scaled boundary finite element method (SBFEM), first proposed by Song and Wolf, is a boundary element method that does not require a fundamental solution. It

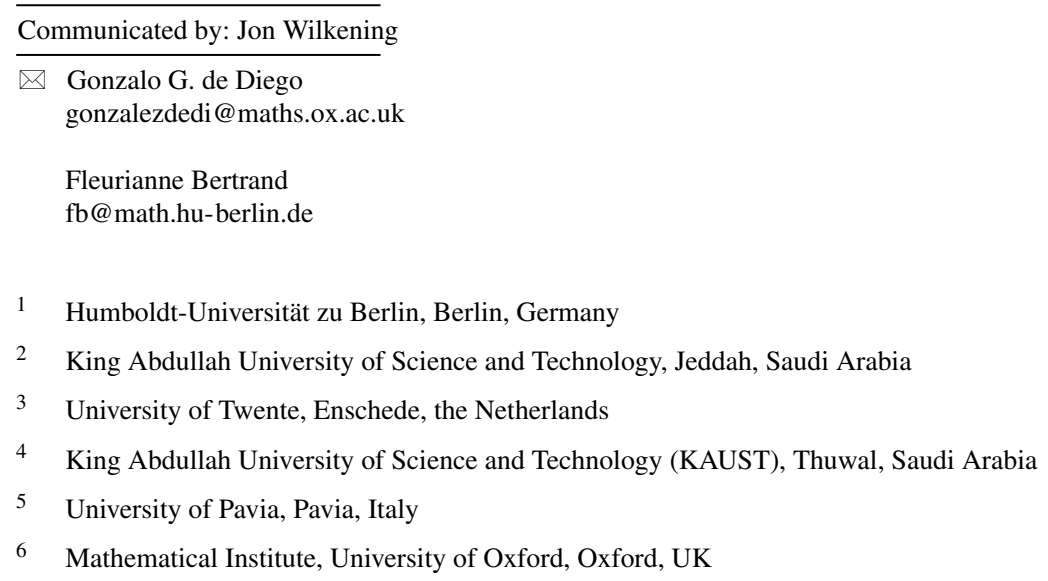


has proven to be particularly effective for problems with singularities or posed over unbounded media, see [6, 7, 9]. In SBFEM, a semi-analytical (or semi-discrete, as we shall call it here) solution to a partial differential equation (PDE) is constructed by transforming the weak formulation of the PDE into an ordinary differential equation (ODE). Essentially, given a star-shaped domain $\Omega \subset \mathbb{R}^{n}$, a coordinate transformation is performed (the scaled boundary transformation) in terms of a radial variable and $n-1$ circumferential variables. Then, an approximate solution is sought in a space of functions discretized solely in the circumferential direction. The resulting weak formulation posed over this space is then transformed into an ODE which, under certain circumstances, can be solved exactly, yielding a semi-analytical approximation of the solution to the PDE.

The SBFEM has been applied to a wide range of problems that arise in science and engineering, such as crack propagation [8] and acoustic-structure interactions [3]. Moreover, the limitation to star-shaped domains has been overcome with the development of scaled boundary polygon elements, in which the domain is broken into arbitrarily shaped polygons and shape functions are constructed over these polygons based on SBFEM [1, 4, 5].

The objective of this paper is to introduce a rigorous framework in which the error of the approximate solution obtained by SBFEM can be estimated. In particular, the notion of a semi-discrete solution to a PDE is formalized by defining a space of semidiscrete functions and constructing an interpolation operator onto this space. Then, given a semi-analytical solution obtained in the framework of SBFEM, estimates of its error can be obtained by bounding the interpolation operator's error using Céa's lemma. We limit the analysis to Poisson's equation posed on a circular domain for simplicity; this setting is appropriate to highlight the main features of our theoretical setting.

The overview of this paper is as follows: in Section 2 we describe the continuous problem together with the polar coordinate change of variables. In Section 3 we introduce a semi-discretization of our problem, where the domain is discretized only in the angular coordinate. It is shown that the semi-discrete solution converges optimally to the continuous solution. Section 4, making use of the semi-discretization, transforms the original problem into an ODE. Finally, two numerical results reported in Section 5 show that the method is performing optimally also in the presence of singularities.

\section{Setting of the problem}

Given an angle $\Theta$ in $(0,2 \pi)$, we are considering the Poisson problem in the following circular sector (see Fig. 1):

$$
\Omega:=\left\{(x, y) \in \mathbb{R}^{2}: 0<x^{2}+y^{2}<1,0<\arctan \left(\frac{y}{x}\right)<\Theta\right\} .
$$

Since we are going to consider a change of variables when defining the scaled boundary method, we denote with $\hat{\bullet}$ (with the hat symbol) quantities defined on $\Omega$ 
Fig. 1 Sector of a disk of angle $\Theta$

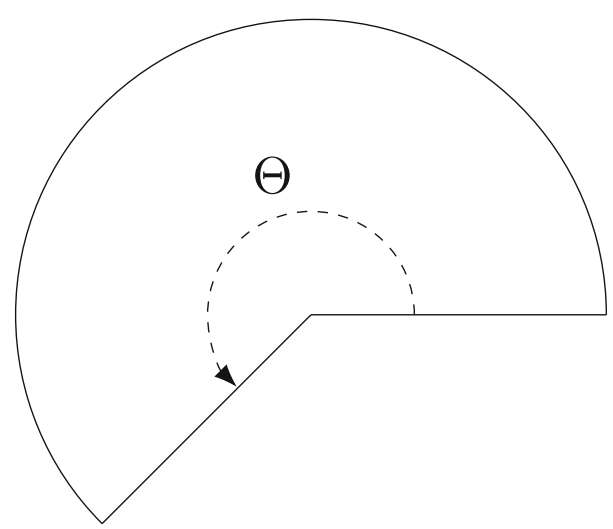

that correspond to quantities $\bullet$ defined on the reference domain. Hence, our problem reads: find $\hat{u}: \Omega \rightarrow \mathbb{R}$ such that

$$
\begin{array}{ll}
-\hat{\Delta} \hat{u}=\hat{f} & \text { in } \Omega \\
\hat{u}=0 & \text { on } \partial \Omega,
\end{array}
$$

where $\hat{f} \in L^{2}(\Omega)$ and $\hat{\Delta}=\partial_{x}^{2}+\partial_{y}^{2}$ is the Laplace operator in the Cartesian coordinates $(x, y)$.

Let the curved part of the boundary of $\Omega$ be parametrized by the graph

$$
\left(x_{b}(\theta), y_{b}(\theta)\right)=(\cos \theta, \sin \theta) \quad \theta \in(0, \Theta)
$$

and define the open rectangle $Q:=(0,1) \times(0, \Theta)$. We consider the mapping $F$ : $Q \rightarrow \mathbb{R}^{2}$ given by

$$
F(r, \theta)=r\left(x_{b}(\theta), y_{b}(\theta)\right) .
$$

In this particular case, the scaled boundary transformation is given by the change of variables $(r, \theta)=F^{-1}(x, y)$ for $(x, y) \in \Omega$, i.e., by the polar coordinate transformation. The Jacobian of $F$ is given by

$$
D F(r, \theta)=\left(\begin{array}{ll}
\partial_{r} x & \partial_{r} y \\
\partial_{\theta} x & \partial_{\theta} y
\end{array}\right)=\left(\begin{array}{cc}
\cos \theta & \sin \theta \\
-r \sin \theta & r \cos \theta
\end{array}\right)
$$

and its determinant is $|D F(r, \theta)|=r$. Since $F$ is differentiable and $|D F(r, \theta)|$ is invertible in the open set $Q$, we have

$$
D F^{-1}(F(r, \theta))=\left(\begin{array}{ll}
\partial_{x} r & \partial_{x} \theta \\
\partial_{y} r & \partial_{y} \theta
\end{array}\right)=\frac{1}{r}\left(\begin{array}{cc}
r \cos \theta & -\sin \theta \\
r \sin \theta & \cos \theta
\end{array}\right) .
$$

Let $u(r, \theta)=\hat{u}(F(r, \theta))$, then the relation between the gradient in Cartesian coordinates $\hat{\nabla}=\left(\partial_{x}, \partial_{y}\right)^{\top}$ and the gradient in polar coordinates $\nabla=\left(\partial_{r}, \partial_{\theta}\right)^{\top}$ is given by

$$
\hat{\nabla} \hat{u}(x, y)=D F^{-1}(x, y) \nabla u\left(F^{-1}(x, y)\right) \quad \text { in } \Omega .
$$

Moreover, the solution $\hat{u}$ of Eq. 1 satisfies

$$
\|\hat{u}\|_{H^{1}(\Omega)}^{2}=\int_{0}^{1} \int_{0}^{\Theta}\left(r u^{2}+r\left(\partial_{r} u\right)^{2}+\frac{1}{r}\left(\partial_{\theta} u\right)^{2}\right) \mathrm{d} r \mathrm{~d} \theta<\infty .
$$


In order to consider the variational formulation of Eq. 1 in polar coordinates, we have to consider appropriate weighted functional spaces. While this is pretty straightforward and well understood, we present the procedure in detail since the notation will be useful for the analysis of the numerical approximation.

Given a weight function $w(r, \theta)$ in $Q$, we define the weighted Lebesgue space

$$
L_{w}^{2}(Q)=\left\{v: Q \rightarrow \mathbb{R} \text { measurable }: \int_{0}^{1} \int_{0}^{\Theta} v^{2} w \mathrm{~d} r \mathrm{~d} \theta<\infty\right\}
$$

with inner product

$$
(u, v)_{L_{w}^{2}(Q)}:=\int_{0}^{1} \int_{0}^{\Theta} u v w \mathrm{~d} r \mathrm{~d} \theta .
$$

We will use in particular $w=r$ and $w=1 / r$; it is not difficult to see that we have $\|u\|_{L_{r}^{2}(Q)} \leq\|u\|_{L^{2}(Q)} \leq\|u\|_{L_{1 / r}^{2}(Q)}$ for all $u \in L_{1 / r}^{2}(Q)$. Furthermore, these spaces are complete [2].

The bound (3) motivates the definition of the following weighted Sobolev space

$$
\tilde{H}^{1}(Q)=\left\{v \in L_{r}^{2}(Q):\|v\|_{L_{r}^{2}(Q)}+\left\|\partial_{r} v\right\|_{L_{r}^{2}(Q)}+\left\|\partial_{\theta} u\right\|_{L_{1 / r}^{2}(Q)}<\infty\right\}
$$

with inner product

$$
(u, v)_{\tilde{H}^{1}(Q)}:=(u, v)_{L_{r}^{2}(Q)}+\left(\partial_{r} u, \partial_{r} v\right)_{L_{r}^{2}(Q)}+\left(\partial_{\theta} u, \partial_{\theta} v\right)_{L_{1 / r}^{2}(Q)} .
$$

The following lemma shows that $H^{1}(\Omega)$ and $\tilde{H}^{1}(Q)$ are isometric.

Lemma 1 Let $\Phi: L^{2}(Q) \rightarrow L_{r}^{2}(Q)$ be defined by $\hat{u} \mapsto \hat{u} \circ F$. Then, the spaces $H^{1}(\Omega)$ and $\tilde{H}^{1}(Q)$ are isometric via $\Phi$.

Proof Let $\hat{u} \in H^{1}(\Omega)$ and, for $0<\rho<1$, let $B_{\rho}$ be the ball of radius $\rho$ centered at the origin and $B_{\rho}^{c}$ its complement. For $\Omega_{\rho}=\Omega \cap B_{\rho}^{c}$, the map $F: Q_{\rho} \rightarrow \Omega_{\rho}$ with $Q_{\rho}:=(\rho, 1) \times(0, \Theta)$ is a bi-Lipschitz map, i.e., there exist two constants $C_{1}, C_{2}>0$ such that

$$
C_{1}\left|\left(r_{1}, \theta_{1}\right)-\left(r_{2}, \theta_{2}\right)\right| \leq\left|F\left(r_{1}, \theta_{1}\right)-F\left(r_{2}, \theta_{2}\right)\right| \leq C_{2}\left|\left(r_{1}, \theta_{1}\right)-\left(r_{2}, \theta_{2}\right)\right|
$$

holds for all $\left(r_{1}, \theta_{1}\right),\left(r_{2}, \theta_{2}\right) \in Q_{\rho}$. Indeed, by the mean value theorem, we have

$$
\left|F\left(r_{1}, \theta_{1}\right)-F\left(r_{2}, \theta_{2}\right)\right| \leq\|\nabla F\|\left|\left(r_{1}, \theta_{1}\right)-\left(r_{2}, \theta_{2}\right)\right|
$$

and clearly $\|\nabla F\|_{\infty} \leq 1$. In the same way, $F^{-1}: \Omega_{\rho} \rightarrow Q_{\rho}$ is a smooth, bijective map and $\left\|F^{-1}\right\|_{\infty} \leq 1 / \rho$; hence, it is Lipschitz continuous and it follows that $F$ and $F^{-1}$ are bi-Lipschitz when restricted to $Q_{\rho}$ and $\Omega_{\rho}$ respectively. As a result of [10, Theorem 2.2.2.], $u=\Phi(\hat{u})$ is weakly differentiable on $Q_{\rho}$ and the chain rule holds. For $n \in \mathbb{N}$, define $u_{n}=\left.\Phi\right|_{\Omega_{1 / n}}(\hat{u})$ on $Q$ by extending $\hat{u}$ by zero outside $\Omega_{\rho}$. For any $0<\rho<1$ one has that

$$
\|u\|_{\tilde{H}^{1}\left(Q_{\rho}\right)}=\|\hat{u}\|_{H^{1}\left(\Omega_{\rho}\right)} \leq\|\hat{u}\|_{H^{1}(\Omega)},
$$


so $u_{n}$ and its derivatives belong to the associated weighted Lebesgue spaces. As a result of the monotone convergence theorem, we have that $u \in \tilde{H}^{1}(Q)$ and

$$
\|u\|_{\tilde{H}^{1}(Q)}=\|\hat{u}\|_{H^{1}(\Omega)} .
$$

Repeating the steps above, we can also show that for $u \in \tilde{H}^{1}(Q)$ one has $\Phi^{-1}(u) \in$ $H^{1}(\Omega)$.

It is then natural to define the following space in order to take into account the boundary conditions

$$
\tilde{H}_{0}^{1}(Q):=\Phi\left(H_{0}^{1}(\Omega)\right) .
$$

We are now ready to state the variational formulation of Eq. 1 in both coordinate systems.

Definition 1 (Weak form of the Poisson problem in Cartesian coordinates) Find $\hat{u} \in$ $H_{0}^{1}(\Omega)$ such that

$$
\hat{a}(\hat{u}, \hat{v})=\hat{b}(\hat{u}) \quad \text { for all } \hat{v} \in H_{0}^{1}(\Omega),
$$

with

$$
\hat{a}(\hat{u}, \hat{v})=(\hat{\nabla} \hat{u}, \hat{\nabla} \hat{v})_{L^{2}(\Omega)}, \quad \hat{b}(\hat{u})=(\hat{f}, \hat{v})_{L^{2}(\Omega)}
$$

Definition 2 (Weak form of the Poisson problem in polar coordinates) Find $u \in$ $\tilde{H}_{0}^{1}(Q)$ such that

$$
a(u, v)=b(v) \quad \text { for all } v \in \tilde{H}_{0}^{1}(Q),
$$

with

$$
\begin{aligned}
a(u, v) & =\int_{0}^{1} \int_{0}^{\Theta}\left(\partial_{r} u \partial_{r} v+\frac{1}{r^{2}} \partial_{\theta} u \partial_{\theta} v\right) r \mathrm{~d} r \mathrm{~d} \theta \\
b(v) & =\int_{0}^{1} \int_{0}^{\Theta} f v r \mathrm{~d} r \mathrm{~d} \theta .
\end{aligned}
$$

It is well-known that Eq. 5 is well posed and so is Eq. 6 thanks to the properties of the map $\Phi$ and of the isometry shown above.

\section{The semi-discrete Poisson equation}

The discretization of Eq. 1 with the scaled boundary finite element method is based on a spatial semi-discretization that is described in this section.

We introduce a partition of the parametrized boundary $\theta \mapsto(\cos \theta, \sin \theta)$ given by

$$
\mathcal{T}_{\Gamma}=\left\{\theta_{1}, \ldots, \theta_{N}\right\}
$$

and consider a finite dimensional approximation of $H^{1}(0, \Theta)$ generated by a basis $\left\{e_{i}(\theta)\right\}_{i=1}^{N}$ with the property that

$$
e_{i}\left(\theta_{j}\right)=\delta_{i j}
$$


Remark 1 The choice of $\left\{e_{i}(\theta)\right\}_{i=1}^{N}$ at this point is arbitrary. It could be based on finite elements, splines, global Lagrange polynomials, etc.

Due to our choice of boundary conditions, we could also have defined the basis $\left\{e_{i}(\theta)\right\}_{i=1}^{N}$ in $H_{0}^{1}(0, \Theta)$, but we prefer to avoid this in order to allow our analysis to be extended more easily to more general boundary conditions or to a situation where $\Theta=2 \pi$.

The main idea behind the semi-discretization is to consider families of functions where the variables $r$ and $\theta$ are separated formally as follows:

$$
u_{S}(r, \theta)=\sum_{i=1}^{N} u_{i}(r) e_{i}(\theta) .
$$

Ideally, we would like to have $u_{i}(r)=u\left(r, \theta_{i}\right)$ and this choice will be used later in Section 3.1 for the error analysis; it will lead to the analogous of the interpolation operator for standard finite elements. In order to do so, we need to give sense to the radial trace $u\left(r, \theta_{i}\right)$. For the sake of readability, we now introduce an abstract setting and we postpone the actual definition of the involved functional spaces to Section 3.1. Ultimately, we want to define a semi-discrete space

$$
U^{s}:=\left\{v_{s} \in \tilde{H}^{1}(Q): v_{s}=\sum_{i=1}^{N} v_{i}(r) e_{i}(\theta) \text { with } v_{i} \in \tilde{U} \text { for } 1 \leq i \leq N\right\},
$$

where $\tilde{U}$ is a suitable functional space on the interval $(0,1)$. We will then consider its subspace $U_{0}^{s}=U^{s} \cap \tilde{H}_{0}^{1}(Q)$, so that the semi-discretization of problem (6) will read: find $u_{s} \in U_{0}^{s}$ such that

$$
a\left(u_{s}, v_{s}\right)=b\left(v_{s}\right) \quad \text { for all } v_{s} \in U_{0}^{s} .
$$

We will prove (see Theorem 1 ) that $U_{0}^{s}$ is a closed subspace of $\tilde{H}_{0}^{1}(Q)$ so that problem (7) is uniquely solvable and the error between $u$ and $u_{s}$ is bounded as usual by the best approximation using Céa's lemma:

$$
\left\|u-u_{s}\right\|_{\tilde{H}^{1}(Q)} \leq C \inf _{v \in U_{0}^{s}}\|u-v\|_{\tilde{H}^{1}(Q)} .
$$

The solution of problem (7) is actually computed by solving a system of ordinary differential equations where the unknowns are the coefficients $u_{i}(r)$ of $u_{s}(r, \theta)$. This procedure is detailed in Section 4.

In order to show the convergence of this procedure, we need to estimate the righthand side of Eq. 8 .

\subsection{Error estimates for the interpolation operator}

We plan to construct an interpolation operator $\Pi$ with values in $U^{s}$ that, if applied to smooth functions, would act as follows

$$
(\Pi u)(r, \theta)=\sum_{i=1}^{N} u\left(r, \theta_{i}\right) e_{i}(\theta) .
$$


Since we will work with Sobolev functions, it is useful to define an adequate tracelike operator that we are going to call the "radial trace operator." To this end, the following bound is required.

Lemma 2 For all $u \in C^{\infty}(\bar{Q})$ and $0 \leq \vartheta \leq \Theta$, we have

$$
\int_{0}^{1} r u^{2}(r, \vartheta) \mathrm{d} r \leq C\left(\|u\|_{L_{r}^{2}(Q)}^{2}+\left\|\partial_{\theta} u\right\|_{L_{r}^{2}(Q)}^{2}\right)
$$

where $C>0$ only depends on $\Theta$.

Proof Let $u \in C^{\infty}(\bar{Q})$ and assume, without loss of generality, that $0 \leq \vartheta<\Theta$. Then, we have

$$
\int_{\vartheta}^{\theta} \partial_{\zeta}\left(u^{2}(r, \zeta)\right) r \mathrm{~d} \zeta=r u^{2}(r, \theta)-r u^{2}(r, \vartheta) \quad \text { for all } \theta \in(\vartheta, \Theta] .
$$

Reordering and integrating over $r$, we have

$$
\int_{0}^{1} r u^{2}(r, \vartheta) \mathrm{d} r=\int_{0}^{1} r u^{2}(r, \theta) \mathrm{d} r-2 \int_{0}^{1} \int_{\vartheta}^{\theta} u(r, \zeta) \partial_{\zeta} u(r, \zeta) r \mathrm{~d} \zeta \mathrm{d} r .
$$

For the last term, we can apply Hölder's inequality, so that

$$
\begin{aligned}
-2 \int_{0}^{1} \int_{\vartheta}^{\theta} u(r, \zeta) \partial_{\zeta} u(r, \zeta) r \mathrm{~d} \zeta \mathrm{d} r & \leq 2 \int_{0}^{1} \int_{0}^{\Theta}\left|u(r, \zeta) \partial_{\zeta} u(r, \zeta)\right| r \mathrm{~d} \zeta \mathrm{d} r \\
& \leq 2\|u\|_{L_{r}^{2}(Q)}\left\|\partial_{\theta} u\right\|_{L_{r}^{2}(Q)} \\
& \leq\|u\|_{L_{r}^{2}(Q)}^{2}+\left\|\partial_{\theta} u\right\|_{L_{r}^{2}(Q)}^{2} .
\end{aligned}
$$

Finally, integrating over $\theta$, we have

$$
\Theta \int_{0}^{1} r u^{2}(r, \vartheta) \mathrm{d} r \leq 2\|u\|_{L_{r}^{2}(Q)}^{2}+\left\|\partial_{\theta} u\right\|_{L_{r}^{2}(Q)}^{2},
$$

so that Eq. 9 holds for smooth functions.

The following space on the interval $(0,1)$ will be used for the definition of $\tilde{U}$

$$
H_{r}^{1}(0,1)=\left\{u \in L_{r}^{2}(0,1): \int_{0}^{1}\left(u^{\prime}(r)\right)^{2} r \mathrm{~d} r<\infty\right\} .
$$

Given an angle $0 \leq \vartheta \leq \Theta$, inequality (9) shows that the natural norm for a space $U$ where the radial trace operator can be defined is

$$
\|u\|_{U}:=\left(\|u\|_{\tilde{H}^{1}(Q)}^{2}+\left\|\partial_{r \theta} u\right\|_{L_{r}^{2}(Q)}^{2}\right)^{\frac{1}{2}} .
$$

It is apparent that not all functions in $C^{\infty}(\bar{Q})$ have a bounded $U$-norm because in general $C^{\infty}(\bar{Q})$ is not included in $\tilde{H}^{1}(Q)$. This is due to the fact that $\left\|\partial_{\theta} u\right\|_{L_{1 / r}^{2}(Q)}$ might not be bounded for some $u \in C^{\infty}(\bar{Q})$. Hence, we define

$$
\tilde{\gamma}_{\vartheta}: C^{\infty}(\bar{Q}) \cap \tilde{H}^{1}(Q) \rightarrow H_{r}^{1}(0,1) \quad u \mapsto u(\cdot, \vartheta)
$$


and extend it to the closure of $C^{\infty}(\bar{Q}) \cap \tilde{H}^{1}(Q)$ with respect to the $U$-norm. We denote by $U \subset \tilde{H}^{1}(Q)$ this space and by $\gamma_{\vartheta}$ the extension of the trace operator, so that we have a bounded radial trace operator

$$
\gamma_{\vartheta}: U \rightarrow H_{r}^{1}(0,1)
$$

that extends the restriction operator $\tilde{\gamma}_{\vartheta}$ defined on smooth enough functions.

It is then natural to choose $\tilde{U}=H_{r}^{1}(0,1)$, so that the definition of $U^{s}$ reads as follows

$$
U^{s}:=\left\{v_{s} \in \tilde{H}^{1}(Q): v_{s}=\sum_{i=1}^{N} v_{i}(r) e_{i}(\theta) \text { with } v_{i} \in H_{r}^{1}(0,1) \text { for } 1 \leq i \leq N\right\}
$$

Theorem 1 The space of semi-discrete functions $U^{s}$ is a closed subspace of $\tilde{H}^{1}(Q)$.

Proof Let $\left(u_{n}\right)$ be a Cauchy sequence in $U^{s}$. By completeness, there is a function $\tilde{u}$ such that $u_{n} \rightarrow \tilde{u}$ in $\tilde{H}^{1}(Q)$. By Lemma 2 we have

$$
\int_{0}^{1} r\left|u_{m}\left(r, \theta_{i}\right)-u_{n}\left(r, \theta_{i}\right)\right|^{2} \mathrm{~d} r \leq C\left\|u_{m}-u_{n}\right\|_{\tilde{H}^{1}(Q)}^{2} \rightarrow 0
$$

as $n, m \rightarrow \infty$, so $\left(u_{n}\left(\cdot, \theta_{i}\right)\right)$ is a Cauchy sequence in $L_{r}^{2}(0,1)$ and by completeness there is a limit $u_{n}\left(\cdot, \theta_{i}\right) \rightarrow u_{i}$ for each $i$. Define $u=\sum_{i=1}^{N} u_{i} e_{i}$ in $U^{s}$ and note that

$$
\begin{aligned}
\|u-\tilde{u}\|_{L_{r}^{2}(Q)} & \leq\left\|u-u_{n}\right\|_{L_{r}^{2}(Q)}+\left\|\tilde{u}-u_{n}\right\|_{L_{r}^{2}(Q)} \\
& \leq C\left(\sum_{i=1}^{N}\left\|u_{i}(\cdot)-u_{n}\left(\cdot, \theta_{i}\right)\right\|_{L_{r}^{2}(0,1)}^{2}\right)^{\frac{1}{2}}+\left\|\tilde{u}-u_{n}\right\|_{L_{r}^{2}(Q)} \rightarrow 0
\end{aligned}
$$

as $n \rightarrow \infty$, so $u=\tilde{u}$ and therefore $u_{n} \rightarrow u$ in $\tilde{H}^{1}(Q)$.

Given $u \in U$ we can then define the interpolant as

$$
(\Pi u)(r, \theta)=\sum_{i=1}^{N} u_{i}(r) e_{i}(\theta),
$$

where $u_{i}(r)$ is defined as $\gamma_{\theta_{i}}(u)$ in $H_{r}^{1}(0,1)$. In order to see that the interpolant is well defined, we need to show that $\Pi u$ belongs to $\tilde{H}^{1}(Q)$. To limit the technicalities, from now on in this section we are assuming that $\left\{e_{i}\right\}$ is the basis of continuous piecewise linear finite elements on $(0, \Theta)$. The general case can be handled with similar arguments.

Lemma 3 For $u \in U$, we have $\Pi u \in \tilde{H}^{1}(Q)$.

Proof We have that $\Pi u \in \tilde{H}^{1}(Q)$ if and only if

$$
\int_{0}^{1} \int_{0}^{2 \pi}\left(r(\Pi u)^{2}+r\left(\partial_{r} \Pi u\right)^{2}+\frac{1}{r}\left(\partial_{\theta} \Pi u\right)^{2}\right) \mathrm{d} \theta \mathrm{d} r<\infty .
$$


We apply Lemma 2 and obtain

$$
\begin{aligned}
\|\Pi u\|_{L_{r}^{2}(Q)}^{2}+\left\|\partial_{r} \Pi u\right\|_{L_{r}^{2}(Q) \leq}^{2} \leq & N \sum_{i=1}^{N}\left(\int_{0}^{1} r u_{i}^{2}(r) \mathrm{d} r \int_{0}^{2 \pi} e_{i}^{2}(\theta) \mathrm{d} \theta\right. \\
& \left.+\int_{0}^{1} r\left(\partial_{r} u_{i}(r)\right)^{2} \mathrm{~d} r \int_{0}^{2 \pi} e_{i}^{2}(\theta) \mathrm{d} \theta\right) \\
\leq & C N\left(\|u\|_{\tilde{H}^{1}(Q)}^{2}+\left\|\partial_{r \theta} u\right\|_{L_{r}^{2}(Q)}^{2}\right) \sum_{i=1}^{N} \int_{0}^{2 \pi} e_{i}^{2}(\theta) \mathrm{d} \theta \\
\leq & \frac{4 \pi^{2} C h}{3 h_{\min }^{2}}\left(\|u\|_{\tilde{H}^{1}(Q)}^{2}+\left\|\partial_{r \theta} u\right\|_{L_{r}^{2}(Q)}^{2}\right),
\end{aligned}
$$

where $h=\max _{i}\left(\theta_{i+1}-\theta_{i}\right)$ and $h_{\min }=\min _{i}\left(\theta_{i+1}-\theta_{i}\right)$.

For the third term in Eq. 10, we fix $r \in(0,1)$ and observe that

$$
u\left(r, \theta_{i}\right)=(\Pi u)\left(r, \theta_{i}\right), \quad u\left(r, \theta_{i+1}\right)=(\Pi u)\left(r, \theta_{i+1}\right)
$$

for $i=1,2, \ldots, N-1$. Taking into account that $\partial_{\theta} \Pi u$ is well defined in $\left(\theta_{i}, \theta_{i+1}\right)$, we apply the mean value theorem and

$$
u\left(r, \theta_{i+1}\right)-u\left(r, \theta_{i}\right)=\left(\theta_{i+1}-\theta_{i}\right)\left(\partial_{\theta} \Pi u(r, \tilde{\theta})\right)
$$

holds for some $\tilde{\theta} \in\left(\theta_{i}, \theta_{i+1}\right)$. Since $\Pi u$ is linear in this interval, the following equality holds

$$
\left|\partial_{\theta} \Pi u(r, \theta)\right|^{2}\left(\theta_{i+1}-\theta_{i}\right)^{2}=\left|\int_{\theta_{i}}^{\theta_{i+1}} \partial_{\theta} u(r, \zeta) \mathrm{d} \zeta\right|^{2}
$$

for all $\theta \in\left(\theta_{i}, \theta_{i+1}\right)$ and $r \in(0,1)$. After multiplying by $1 / r$, integrating, and applying Hölder's inequality, we have

$$
\int_{0}^{1} \int_{\theta_{i}}^{\theta_{i+1}} \frac{1}{r}\left|\partial_{\theta} \Pi u(r, \theta)\right|^{2} \mathrm{~d} \theta \mathrm{d} r \leq \int_{0}^{1} \int_{\theta_{i}}^{\theta_{i+1}} \frac{1}{r}\left|\partial_{\theta} u(r, \theta)\right|^{2} \mathrm{~d} \theta \mathrm{d} r .
$$

By integrating over each interval and summing up the terms, we have

$$
\left\|\partial_{\theta} \Pi u\right\|_{L_{1 / r}^{2}(Q)} \leq\left\|\partial_{\theta} u\right\|_{L_{1 / r}^{2}(Q)} .
$$

Finally, putting (11) and (12) together, we get

$$
\|\Pi u\|_{\tilde{H}^{1}(Q)}^{2} \leq \max \left\{\frac{4 \pi^{2} C h}{3 h_{\min }^{2}}, 1\right\}\left(2\|u\|_{\tilde{H}^{1}(Q)}^{2}+\left\|\partial_{r \theta} u\right\|_{L_{r}^{2}(Q)}^{2}\right)<\infty .
$$

In the next theorem, we prove the approximation properties of $U^{s}$. As usual, we need to assume suitable regularity that will be characterized by the following space

$$
U^{\prime}=\left\{u \in U:\left\|\partial_{\theta \theta} u\right\|_{L_{1 / r}^{2}(Q)}<\infty\right\} .
$$


Remark 2 The space $U^{\prime}$ requires extra regularity only in the angular variable $\theta$. We will see in Section 5 that singular solutions (with respect to the Cartesian coordinates) can be in $U^{\prime}$ and be approximated optimally by SBFEM.

Theorem 2 Let $u$ be in $U^{\prime}$. Then, there exists $C>0$ independent of $\mathcal{T}_{\Gamma}$ such that

$$
\|u-\Pi u\|_{L_{r}^{2}(Q)} \leq h^{2}\left\|\partial_{\theta \theta} u\right\|_{L_{1 / r}^{2}(Q)}
$$

and

$$
\|u-\Pi u\|_{\tilde{H}^{1}(Q)} \leq C h\left(\left\|\partial_{r \theta} u\right\|_{L_{r}^{2}(Q)}^{2}+\left\|\partial_{\theta \theta} u\right\|_{L_{1 / r}^{2}(Q)}^{2}\right) .
$$

Proof Let $u \in C^{\infty}(\bar{Q})$ and define $\varepsilon(r, \theta)=(u-\Pi u)(r, \theta)$. Due to the properties of the interpolation operator, we have $\varepsilon\left(r, \theta_{i}\right)=0$ for $i=1, \ldots, N$. As a result, there is a $\tilde{\theta}_{i} \in\left(\theta_{i}, \theta_{i+1}\right)$ such that $\partial_{\theta} \varepsilon\left(r, \tilde{\theta}_{i}\right)=0$. It follows that

$$
\partial_{\theta} \varepsilon(r, \vartheta)=\int_{\tilde{\theta}_{i}}^{\vartheta} \partial_{\theta \theta} \varepsilon(r, \zeta) \mathrm{d} \zeta=\int_{\tilde{\theta}_{i}}^{\vartheta} \partial_{\theta \theta} u(r, \zeta) \mathrm{d} \zeta \quad \text { for } \tilde{\theta}_{i}<\vartheta \leq \theta_{i+1},
$$

since $\Pi u$ is linear in $\left(\theta_{i}, \theta_{i+1}\right)$ in the $\theta$ direction. Applying Hölder's inequality we have

$$
\left|\partial_{\theta} \varepsilon(r, \vartheta)\right|^{2} \leq\left(\theta_{i+1}-\theta_{i}\right) \int_{\theta_{i}}^{\theta_{i+1}}\left|\partial_{\theta \theta} u(r, \theta)\right|^{2} \mathrm{~d} \theta .
$$

Integrating over the domain and summing up the different terms corresponding to each interval $\left(\theta_{i}, \theta_{i+1}\right)$ we have

$$
\left\|\partial_{\theta} \varepsilon\right\|_{L_{1 / r}(Q)}^{2} \leq h^{2}\left\|\partial_{\theta \theta} u\right\|_{L_{1 / r}(Q)}^{2} .
$$

Since both $\|\cdot\|_{L_{1 / r}(Q)}$ and $\Pi$ are continuous, inequality (13) can be shown to hold for all $u \in U^{\prime}$ by a density argument. Likewise, for $\varepsilon(r, \vartheta)$ we have

$$
|\varepsilon(r, \vartheta)|^{2}=\left|\int_{\theta_{i}}^{\vartheta} \partial_{\theta} \varepsilon(r, \zeta) \mathrm{d} \zeta\right|^{2} \leq h \int_{\theta_{i}}^{\theta_{i+1}}\left|\partial_{\theta} \varepsilon(r, \zeta)\right|^{2} \mathrm{~d} \zeta
$$

After integrating and using Eq. 13, we have

$$
\|\varepsilon\|_{L_{r}^{2}(Q)}^{2} \leq h^{4}\left\|\partial_{\theta \theta} u\right\|_{L_{1 / r}^{2}(Q)}^{2} .
$$

Finally, an estimate must be found for $\left\|\partial_{r} \varepsilon\right\|_{L_{r}^{2}(Q)}$. Once again, we consider a smooth function $u$ and take into account that $\partial_{r} \varepsilon\left(r, \theta_{i}\right)=0$ for all $r \in(0,1)$ and $i=1, \ldots, N$. Hence,

and it follows that

$$
\partial_{r} \varepsilon(r, \vartheta)=\int_{\theta_{i}}^{\vartheta} \partial_{r \theta} \varepsilon(r, \zeta) \mathrm{d} \zeta
$$

$$
\left\|\partial_{r} \varepsilon\right\|_{L_{r}^{2}(Q)}^{2} \leq h^{2}\left\|\partial_{r \theta} \varepsilon\right\|_{L_{1 / r}^{2}(Q)}^{2} .
$$

In the same way as Eq. 12 is obtained, we apply the mean value theorem to the function $\partial_{r}(\Pi u)(r, \vartheta)$ for $\vartheta \in\left(\theta_{i}, \theta_{i+1}\right)$ and obtain

$$
\left(\partial_{r \theta}(\Pi u)(r, \vartheta)\right)\left(\theta_{i+1}-\theta_{i}\right)=\int_{\theta_{i}}^{\theta_{i+1}} \partial_{r \theta} u(r, \zeta) \mathrm{d} \zeta
$$


and therefore we can establish that

$$
\left\|\partial_{r \theta} \Pi u\right\|_{L_{r}^{2}(Q)} \leq\left\|\partial_{r \theta} u\right\|_{L_{r}^{2}(Q)} .
$$

Given (16), we have the following error estimate for all smooth functions $u$ :

$$
\begin{aligned}
\left\|\partial_{r} \varepsilon\right\|_{L_{r}^{2}(Q)}^{2} & \leq 2 h^{2}\left(\left\|\partial_{r \theta} u\right\|_{L_{r}^{2}(Q)}^{2}+\left\|\partial_{r \theta} \Pi u\right\|_{L_{r}^{2}(Q)}^{2}\right) \\
& \leq 4 h^{2}\left\|\partial_{r \theta} u\right\|_{L_{r}^{2}(Q)}^{2}
\end{aligned}
$$

which, by a density argument, holds for all $u \in U^{\prime}$. Therefore, putting (13), (14), and (17) together, we obtain the required bound. More precisely, we have

$$
\|u-\Pi u\|_{\tilde{H}^{1}(Q)}^{2} \leq h^{2}\left(h^{2}+4\right)\left(\left\|\partial_{r \theta} u\right\|_{L_{r}^{2}(Q)}^{2}+\left\|\partial_{\theta \theta} u\right\|_{L_{1 / r}(Q)}^{2}\right) .
$$

\section{Constructing semi-discrete solutions with SBFEM}

In order to solve our problem, the scaled boundary finite element method rewrites the formulation (7) as a system of ordinary differential equations; this is carried out in Section 4.1. This is possible thanks to the representation of semi-discrete functions in $U_{0}^{S}$ as the product of $r$-dependent functions $u_{i}(r)$ with $\theta$-dependent test functions. The resulting system of differential equations is supplemented with additional conditions arising from regularity requirements and the boundary conditions. This system can be solved by an analytical method under certain conditions, a process which is described in Section 4.2.

\subsection{Rewriting the Poisson equation as an ODE}

Let us consider $u_{s}=\sum_{i=1}^{N} u_{i} e_{i}$ and $v_{s}=\sum_{i=1}^{N} v_{i} e_{i}$. An immediate consequence of the definition of the space of semi-discrete functions $U_{0}^{s}$ is that the bilinear form $a: U_{0}^{s} \times U_{0}^{s} \rightarrow \mathbb{R}$ and the linear form $b: U_{0}^{s} \rightarrow \mathbb{R}$ may be rewritten as follows:

$$
\begin{aligned}
a\left(u_{s}, v_{s}\right)= & \sum_{i, j=1}^{N} \int_{0}^{1} \int_{0}^{\Theta}\left(u_{i}^{\prime}(r) v_{j}^{\prime}(r) e_{i}(\theta) e_{j}(\theta)+\frac{1}{r^{2}} u_{i}(r) v_{j}(r) e_{i}^{\prime}(\theta) e_{j}^{\prime}(\theta)\right) r \mathrm{~d} r \mathrm{~d} \theta \\
= & \sum_{i, j=1}^{N}\left(\int_{0}^{1} u_{i}^{\prime}(r) v_{j}^{\prime}(r) r \mathrm{~d} r \int_{0}^{\Theta} e_{i}(\theta) e_{j}(\theta) \mathrm{d} \theta\right. \\
& \left.+\int_{0}^{1} u_{i}(r) v_{j}(r) \frac{\mathrm{d} r}{r} \int_{0}^{\Theta} e_{i}^{\prime}(\theta) e_{j}^{\prime}(\theta) \mathrm{d} \theta\right) \\
= & \sum_{i, j=1}^{n}\left(\mathrm{~A}_{i j} \int_{0}^{1} u_{i}^{\prime}(r) v_{j}^{\prime}(r) r \mathrm{~d} r+\mathrm{B}_{i j} \int_{0}^{1} u_{i}(r) v_{j}(r) \frac{\mathrm{d} r}{r}\right)
\end{aligned}
$$


and

$$
\begin{aligned}
b\left(v_{s}\right) & =\sum_{j=1}^{N} \int_{0}^{1} \int_{0}^{\Theta} f(r, \theta) v_{j}(r) e_{j}(\theta) r \mathrm{~d} r \mathrm{~d} \theta \\
& =\sum_{j=1}^{N} \int_{0}^{1} F_{j}(r) v_{j}(r) r \mathrm{~d} r,
\end{aligned}
$$

where

$$
\begin{aligned}
& \mathrm{A}_{i j}=\int_{0}^{\Theta} e_{i}(\theta) e_{j}(\theta) \mathrm{d} \theta \\
& \mathrm{B}_{i j}=\int_{0}^{\Theta} e_{i}^{\prime}(\theta) e_{j}^{\prime}(\theta) \mathrm{d} \theta \\
& F_{j}(r)=\int_{0}^{\Theta} f(r, \theta) e_{j}(\theta) \mathrm{d} \theta .
\end{aligned}
$$

We now proceed formally with the derivation of the differential equation. To this aim, we will use the following integration by parts formula

$$
\int_{0}^{1} u_{i}^{\prime}(r) v_{j}^{\prime}(r) r \mathrm{~d} r=-\int_{0}^{1} u_{i}^{\prime \prime}(r) v_{j}(r) r \mathrm{~d} r-\int_{0}^{1} u_{i}^{\prime}(r) v_{j}(r) \mathrm{d} r+u_{i}^{\prime}(1) v_{j}(1)
$$

which is clearly valid for smooth enough $u_{i}$ and $v_{i}$.

In order to simplify our notation, we introduce a name for the space of the coefficients in $U^{s}$ as follows

$$
\boldsymbol{U}_{0}^{s}=\left\{\boldsymbol{u}=\left(u_{i}\right)_{i=1}^{N}: u_{i} \in H_{r}^{1}(0,1) \text { for } 1 \leq i \leq N, \sum_{i=1}^{N} u_{i} e_{i} \in U_{0}^{s}\right\} .
$$

It follows that if $u_{s} \in U_{0}^{s}$ is smooth enough, it solves $a\left(u_{s}, v_{s}\right)=b\left(v_{s}\right)$ for all $v_{s} \in U_{0}^{s}$ if and only if

$$
\sum_{j=1}^{N} \int_{0}^{1} v_{j}(r) \sum_{i=1}^{N}\left[-r \mathrm{~A}_{i j} u_{i}^{\prime \prime}(r)-\mathrm{A}_{i j} u_{i}^{\prime}(r)+\frac{1}{r} \mathrm{~B}_{i j} u_{i}(r)-F_{j}(r) r\right] \mathrm{d} r=0
$$

holds for all $\boldsymbol{v}=\left(v_{i}\right)_{i=1}^{N} \in \boldsymbol{U}_{0}^{s}$. Moreover, as a result of the fundamental lemma of calculus of variations, (18) holds if and only if

$$
\sum_{j=1}^{N}\left(r \mathrm{~A}_{i j} u_{j}^{\prime \prime}(r)+\mathrm{A}_{i j} u_{j}^{\prime}(r)-\frac{1}{r} \mathrm{~B}_{i j} u_{j}(r)\right)=r F_{i}(r) \text { for a.e. } r \in(0,1)
$$

is satisfied for all $i=2, \ldots, N-1$, since $v_{1}=v_{N}=0$ by $\boldsymbol{v} \in \boldsymbol{U}_{0}^{s}$. Because we seek a solution $\boldsymbol{u} \in \boldsymbol{U}_{0}^{s}$, we must also enforce the boundary conditions $u_{1}=u_{N}=0$ and $u_{i}(1)=0$ for $i=2, \ldots, N-1$. Furthermore, we impose the compatibility condition $u_{i}(0)=u_{j}(0)$ for all $i, j=1, \ldots, N$ to avoid a singularity at the center of the disk. In this, we guarantee that the function $u=\sum_{i=1}^{N} u_{i} e_{i}$ belongs to $\tilde{H}_{0}^{1}(Q)$.

Denote by $\mathbf{A}$ and $\mathbf{B}$ the matrices in $\mathbb{R}^{N \times N}$ with components $A_{i j}$ and $B_{i j}$ and by $\boldsymbol{F}(r)$ the vector function with components $F_{i}(r)$. We modify the columns and rows in $\mathbf{A}$ and $\mathbf{B}$ and we set $F_{i}(r)=0$ for $i=1$ and $N$ in order to enforce $u_{1}=u_{N}=0$. 
Then, a semi-discrete solution of the Poisson equation $\boldsymbol{u}=\left(u_{i}\right)_{i=1}^{N}$ will satisfy the following conditions:

$$
\begin{cases}r^{2} \mathbf{A} \boldsymbol{u}^{\prime \prime}+r \mathbf{A} \boldsymbol{u}^{\prime}-\mathbf{B} \boldsymbol{u}=r^{2} \boldsymbol{F} & \text { for } r \in(0,1), \\ \boldsymbol{u}(1)=0, & \text { for all } i, j=1, \ldots, N . \\ u_{i}(0)=u_{j}(0) & \end{cases}
$$

SBFEM provides a methodology for the construction of solutions for Eq. 20, see the next section. Whenever such solutions exist and satisfy the integration by parts formula for all $\boldsymbol{v} \in \boldsymbol{U}_{0}^{s}$, then they correspond with the unique solution in $U_{0}^{s}$.

\subsection{Solving the ODE analytically}

Following [9], a solution to Eq. 20 can be constructed by finding a family of functions that satisfy the homogeneous ODE together with a particular solution. In order to construct the homogeneous solution, we shall define the matrix $\mathbf{E} \in \mathbb{R}^{2 N \times 2 N}$ by

$$
\mathbf{E}=\left(\begin{array}{cc}
0 & \mathbf{A}^{-1} \\
\mathbf{B} & 0
\end{array}\right)
$$

Note that $\mathbf{E}$ arises when the ODE in Eq. 20 is rewritten as a first-order differential equation by introducing the additional variable $\boldsymbol{q}(r)=r \mathbf{A} \boldsymbol{u}^{\prime}(r)$. If $\left(\lambda,(\boldsymbol{\phi}, \boldsymbol{\psi})^{T}\right)$ is an eigenpair of $\mathbf{E}$, with $\boldsymbol{\phi}, \boldsymbol{\psi} \in \mathbb{C}^{N}$, we can see that

$$
\mathbf{B} \boldsymbol{\phi}=\lambda^{2} \mathbf{A} \boldsymbol{\phi} .
$$

Furthermore, for $\boldsymbol{u}(r)=r^{\lambda} \boldsymbol{\phi}$ we have that

$$
r^{2} \mathbf{A} \boldsymbol{u}^{\prime \prime}+r \mathbf{A} \boldsymbol{u}^{\prime}-\mathbf{B} \boldsymbol{u}=0
$$

for all $r>0$. That is, $\boldsymbol{u}(r)=r^{\lambda} \boldsymbol{\phi}$ is a homogeneous solution of the ODE in Eq. 20 . This idea can be extended in such a way that we get homogeneous solutions as a linear combination of $N$ linearly independent functions that satisfy (22). Indeed, first note that $\mathbf{E}$ is a Hamiltonian matrix and therefore, for every eigenvalue $\lambda \in \mathbb{C}$, we will also have that $-\lambda, \bar{\lambda}$, and $-\bar{\lambda}$ are eigenvalues of $\mathbf{E}$. Consider the following subset of $N$ eigenpairs of $\mathbf{E}$ :

$$
\left\{\left(\lambda_{i},\left(\begin{array}{c}
\boldsymbol{\phi}_{i} \\
\boldsymbol{\psi}_{i}
\end{array}\right)\right): \operatorname{Re}\left(\lambda_{i}\right) \geq 0, \quad \boldsymbol{\phi}_{i}, \boldsymbol{\psi}_{i} \in \mathbb{C}^{N}, \quad i=1, \ldots, N\right\} .
$$

From Eq. 21, we see that the pair $\left(\lambda_{i}^{2}, \boldsymbol{\phi}_{i}\right)$ is an eigenpair of the one-dimensional Laplace problem discretized with piecewise polynomials. As a result, $\lambda_{i} \in \mathbb{R}$ and $\boldsymbol{\phi}_{i} \in \mathbb{R}^{N}$. Moreover, the vectors $\left(\boldsymbol{\phi}_{i}\right)$ form a basis of $\mathbb{R}^{N}$. Then, for any $N$-tuple of real numbers $c_{1}, \ldots, c_{N}$, we have that

$$
\boldsymbol{u}_{h}(r)=\sum_{i=1}^{N} c_{i} r^{\lambda_{i}} \boldsymbol{\phi}_{i}
$$

is a homogeneous solution to the ODE in Eq. 20. Given a particular solution $\boldsymbol{u}_{p}$ for which

$$
r^{2} \mathbf{A} \boldsymbol{u}_{p}^{\prime \prime}+r \mathbf{A} \boldsymbol{u}_{p}^{\prime}-\mathbf{B} \boldsymbol{u}_{p}=r^{2} \boldsymbol{F} \quad \text { on }(0,1)
$$


the constants $c_{1}, \ldots c_{N}$ are then set by enforcing the boundary conditions $\boldsymbol{u}_{h}(1)+$ $\boldsymbol{u}_{p}(1)=0$. The resulting function $\boldsymbol{u}=\boldsymbol{u}_{h}+\boldsymbol{u}_{p}$ is a solution of Eq. 20 .

Remark 3 In the construction of $\boldsymbol{u}$, we only consider non-negative eigenvalues because we require $u_{i}(r) \in H_{r}^{1}(0,1)$ for all $i=1, \ldots, N$. Moreover, the condition $u_{i}(0)=u_{j}(0)$ for all $i, j=1, \ldots, N$ holds because if $\lambda_{i}=0$ then $\phi$ is in the kernel of $\mathbf{B}$, which consists of vectors whose entries are all equal.

The analytical construction of a particular solution relies on the form of $\boldsymbol{F}(r)$. For example, if $\boldsymbol{F}(r)=r^{\alpha} \boldsymbol{f}$, where $\boldsymbol{f} \in \mathbb{R}^{N}$ and $\alpha \in \mathbb{R}$, then it is simple to see that $\boldsymbol{u}_{p}(r)=r^{\alpha+2} \boldsymbol{\phi}_{p}$, with $\boldsymbol{\phi}_{p} \in \mathbb{R}^{N}$ solving the linear system

$$
\left((\alpha+2)^{2} \mathbf{A}-\mathbf{B}\right) \boldsymbol{\phi}_{p}=\boldsymbol{f}
$$

is a particular solution of Eq. 19.

\section{Numerical examples}

One of the main features of the method presented in the previous sections is that it can achieve high order of convergence also in the presence of singular solutions. We are going to show this behavior with two simple examples. The approximate solutions to Eq. 19 are obtained by means of the analytical method described in Section 4.2. In these tests, the basis functions $e_{i}$ in the angular direction are piecewise polynomials of order 1 or higher.

\subsection{A first numerical test}

We take $\Theta=3 \pi / 2$ and consider the function

$$
u_{e}(r, \theta)=r^{\frac{2}{3}} \sin \left(\frac{2}{3} \theta\right) .
$$

This function satisfies $\Delta \hat{u}_{e}=0$ on $\Omega$, where $\hat{u}_{e}=\Phi^{-1}\left(u_{e}\right)$, so it is a solution to the homogeneous problem: find $\hat{u} \in H^{1}(\Omega)$ such that

$$
\begin{aligned}
-\Delta u & =0 & & \text { in } \Omega \\
\hat{u} & =\hat{u}_{e} & & \text { on } \partial \Omega .
\end{aligned}
$$

Our theory can be easily extended to accommodate non-homogeneous boundary conditions. Moreover, it is well-known that $u_{e}$ belongs to $H^{1}(\Omega)$ but not to $H^{2}(\Omega)$. Indeed, this follows from the fact that the following inequality holds for any $0<$ $R<1$ and $0<\varepsilon<R$

$$
\begin{aligned}
\left\|\hat{u}_{e}\right\|_{2, \Omega}^{2} & \geq \int_{\varepsilon}^{R} \int_{0}^{\frac{3}{2} \pi}\left(\frac{\partial u_{e}}{\partial r}\right)^{2} r \mathrm{~d} \theta \mathrm{d} r=\left(\frac{4}{81} \int_{0}^{\frac{3}{2} \pi} \sin ^{2}\left(\frac{2}{3} \theta\right) \mathrm{d} \theta\right) \int_{\varepsilon}^{R} r^{-\frac{5}{3}} \mathrm{~d} r \\
& =C\left(\varepsilon^{-\frac{2}{3}}-R^{-\frac{2}{3}}\right)
\end{aligned}
$$



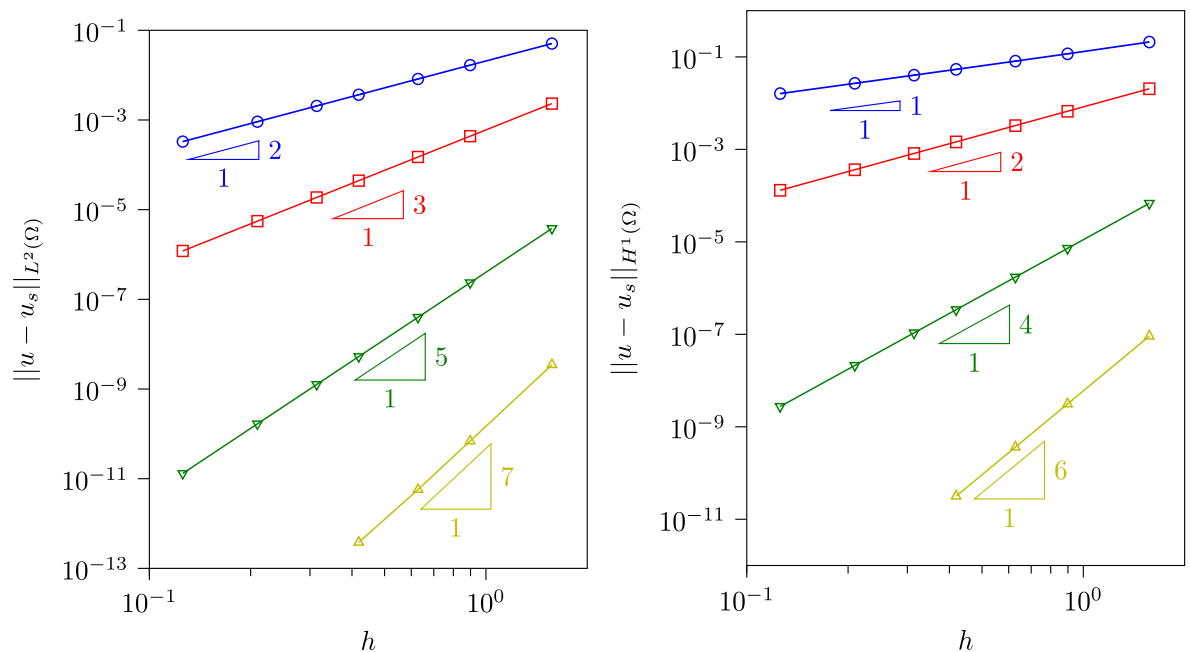

Fig. 2 Convergence plots of $L^{2}$ and $H^{1}$ errors for the first numerical test with basis functions $e_{i}$ of polynomial orders $1,2,4$, and 6

which tends to infinity as $\varepsilon$ goes to 0 . On the other hand, it can be easily seen that $u_{e}$ belongs to $U^{\prime}$ and this makes it possible to use the result of Theorem 2 which implies, in particular, that first-order elements achieve second order of convergence in $L^{2}$ even in presence of a corner singularity.

We report in Fig. 2 the results of our numerical test that confirm our theoretical findings. We include also higher order approximations (up to order six) and a convergence plot in $H^{1}$.
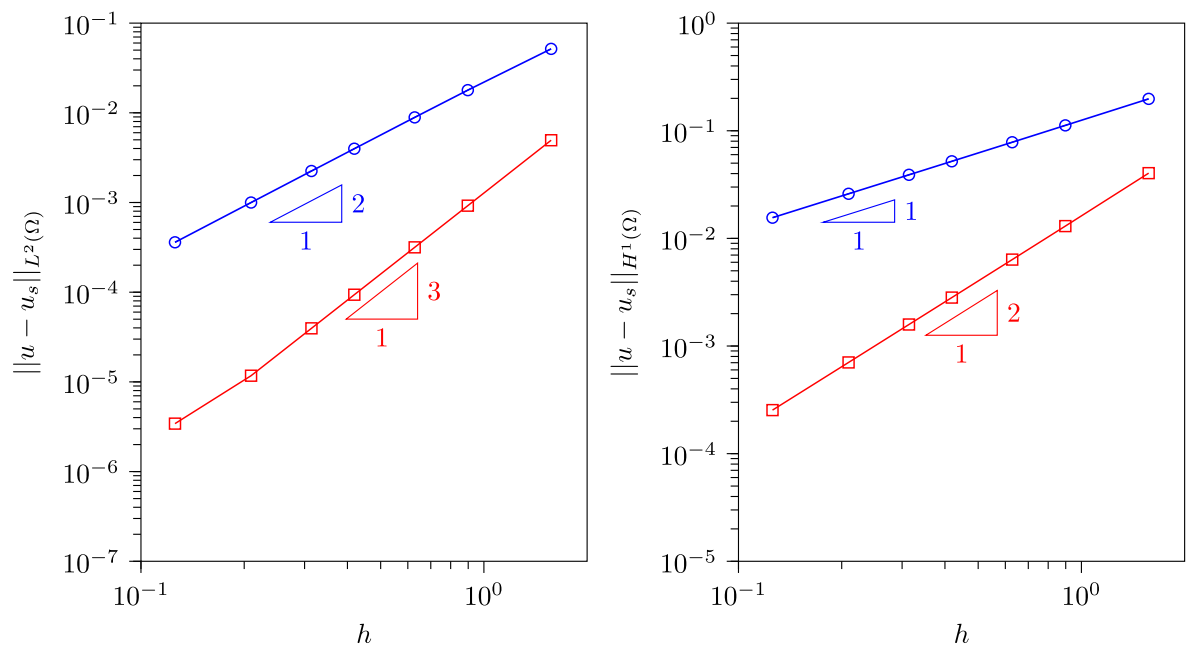

Fig. 3 Convergence plots of $L^{2}$ and $H^{1}$ errors for the second numerical test with basis functions $e_{i}$ of polynomial orders 1 and 2 


\subsection{A second numerical test}

For the second numerical test, we consider a slightly more complicated example. Once again, we set $\Theta=3 \pi / 2$ and consider the function

$$
v_{e}(r, \theta)=r^{\frac{2}{3}}\left(\left(1-\frac{4 \theta}{3 \pi}\right) \cos (2 \theta / 3)-\frac{4}{3 \pi} \log (r) \sin (2 \theta / 3)\right) .
$$

For this function we once again have that $\Delta \hat{v}_{e}=0$, where $\hat{v}_{e}=\Phi^{-1}\left(v_{e}\right)$, and $v_{e}=r^{2 / 3}$ at $\theta=0$ and $\theta=\Theta$. This function therefore is a solution to the problem: find $\hat{u} \in H^{1}(\Omega)$ such that

$$
\begin{aligned}
-\Delta \hat{u} & =0 & & \text { in } \Omega \\
\hat{u} & =\hat{v}_{e} & & \text { on } \partial \Omega .
\end{aligned}
$$

The results to our numerical tests are presented in Fig. 3 for polynomial orders 1 and 2. The resulting convergence rates confirm the theoretical predictions from Section 3.

Acknowledgements We would like to thank the project partners Prof. Carolin Birk (Universität DuisburgEssen, Germany) and Prof. Christian Meyer (TU Dortmund, Germany) as well as Professor Gerhard Starke for the fruitful discussions.

Funding The first author was supported by the German Research Foundation (DFG) in the Priority Programme SPP 1748 Reliable simulation techniques in solid mechanics under grant number BE6511/1-1. The second author is a member of the INdAM Research group GNCS and his research is partially supported by IMATI/CNR and by PRIN/MIUR. The first and third authors were supported by Mercator Research Center Ruhr (MERCUR) under grant Pr-2017-0017.

Open Access This article is licensed under a Creative Commons Attribution 4.0 International License, which permits use, sharing, adaptation, distribution and reproduction in any medium or format, as long as you give appropriate credit to the original author(s) and the source, provide a link to the Creative Commons licence, and indicate if changes were made. The images or other third party material in this article are included in the article's Creative Commons licence, unless indicated otherwise in a credit line to the material. If material is not included in the article's Creative Commons licence and your intended use is not permitted by statutory regulation or exceeds the permitted use, you will need to obtain permission directly from the copyright holder. To view a copy of this licence, visit http://creativecommons.org/licenses/by/4. $0 \%$

\section{References}

1. Gravenkamp, H., Saputra, A.A., Song, C., Birk, C.: Efficient wave propagation simulation on quadtree meshes using SBFEM with reduced modal basis. Int. J. Numer. Methods Eng. 110(12), 1119-1141 (2016)

2. Kufner, A.: Weighted Sobolev Spaces. Teubner-Texte zur Mathematik. B.G. Teubner, Berlin (1985)

3. Liu, L., Zhang, J., Song, C., Birk, C., Saputra, A.A., Gao, W.: Automatic three-dimensional acousticstructure interaction analysis using the scaled boundary finite element method. J. Comput. Phys. 395, 432-460 (2019)

4. Ooi, E.T., Song, C., Tin-Loi, F., Yang, Z.: Polygon scaled boundary finite elements for crack propagation modelling. Int. J. Numer. Methods Eng. 91(3), 319-342 (2012)

5. Ooi, E.T., Song, C., Tin-Loi, F.: A scaled boundary polygon formulation for elasto-plastic analyses. Comput. Methods Appl. Mech. Eng. 268, 905-937 (2014)

6. Song, C., Wolf, J.P.: The scaled boundary finite-element method-alias consistent infinitesimal finiteelement cell method-for elastodynamics. Comput. Methods Appl. Mech. Eng. 147(3), 329-355 (1997) 
7. Song, C., Wolf, J.P.: The scaled boundary finite element method-alias consistent infinitesimal finite element cell method-for diffusion. Int. J. Numer. Methods Eng. 45(10), 1403-1431 (1999)

8. Song, C., Wolf, J.P.: Semi-analytical representation of stress singularities as occurring in cracks in anisotropic multi-materials with the scaled boundary finite-element method. Comput. Struct. 80(2), 183-197 (2002)

9. Wolf, J.: Scaled boundary finite element method. Wiley, New York (2003)

10. Ziemer, W.P.: Weakly differentiable functions. Springer, Berlin (1989)

Publisher's note Springer Nature remains neutral with regard to jurisdictional claims in published maps and institutional affiliations. 